\title{
Tra sintesi vocale e divizzazione olografica Alcune considerazioni sul caso Hatsune Miku
}

\author{
Maël Guesdon - Philippe Le Guern
}

\begin{abstract}
Developed and distributed in 2007 to promote the second version of the Vocaloid software, Hatsune Miku has quickly become a protean phenomenon. First and foremost, Hatsune Miku is a synthetic voice system that the spectator-listener can utilise to create and share custom compositions. Secondly, she is also a holographic celebrity performer appearing in live concerts, and the heroine of a video game series project DIVA. Thirdly, she is the protagonist of numerous fan fiction titles. Finally, since 2013, she is also the lead singer of an opera piece. In this paper, we focus on the Hatsune Miku case, an outstanding example of holography in pop music, which radicalise the question of fan attachment and celebrity, at the crossroads of technology, newly emergent economic models within cultural industries, fan culture and creative audiences.
\end{abstract}

Keywords: Hatsune Miku, Hologram, Popular music, Cultural industries, Fan culture, Capitalism, Innovation. 


\section{Sommario}

Sviluppato e distribuito nel 2007 per promuovere la seconda versione del software Vocaloid, Hatsune Miku è diventato rapidamente un fenomeno proteiforme. Innanzitutto, Hatsune Miku è un sistema di voce sintetica che lo spettatore-ascoltatore può utilizzare per creare e condividere composizioni personalizzate. In secondo luogo, è una celebrità olografica che appare in concerti dal vivo, nonché l'eroina di una serie di videogiochi project DIVA. In terzo luogo, è la protagonista di numerosi titoli di fan fiction. Infine, dal 2013, è anche la cantante principale di un'opera. In questo articolo ci concentriamo sul caso Hatsune Miku, un esempio eccezionale di olografia nella musica pop, che radicalizza la questione dell'attaccamento dei fan e della celebrità, all'incrocio tra tecnologia, nuovi modelli economici emergenti all'interno delle industrie culturali, cultura dei fan e pubblico creativo.

Parole chiave: Hatsune Miku, Ologramma, Musica popolare, Industrie culturali, Cultura dei fan, Capitalismo, Innovazione.

Elaborata e diffusa nel 2007 per promuovere la seconda versione del software Vocaloid, Hatsune Miku è rapidamente diventata un fenomeno proteiforme nel suo presentarsi, di volta in volta, come sintesi vocale di cui lo spettatoreascoltatore può appropriarsi per creare e poi diffondere sul web le proprie composizioni ${ }^{1}$; star olografica che si esibisce in concerti relativamente rari e ricercati; eroina di videogiochi nella serie project $D I V A^{2}$, dove interpreta

\footnotetext{
* Un sentito ringraziamento al dott. Nicolò Palazzetti dell'Université de Strasbourg per i preziosi consigli e l'attenta revisione alla presente traduzione (N.d.T.)

${ }^{1}$ Per poco più di un centinaio di euro, gli utenti possono produrre brani nei quali far cantare Hatsune Miku. Essendo il suo corpo in 3D simulato, e quindi "animabile", questi brani vengono spesso accompagnati da clip video, di solito concepite in collaborazione con altri utenti, e poi postate sui social media o su siti specializzati, nei quali tali prodotti vengono valutati, classificati e commentati da una comunità di fan.

${ }^{2}$ Hatsune Miku: project Diva è stato sviluppato da Sega in collaborazione con Crypton Future Media. Si tratta di un videogioco detto "di rytHatsune Mikue", che consiste nel premere i tasti del controller seguendo le indicazioni che compaiono in sovraimpressione sulla coreografia di Hatsune Miku. Alla base del gioco c'è dunque un brano sul quale Hatsune
} 
alcuni dei suoi successi; personaggio centrale di numerose fan fictions, sui cui elementi narrativi si basano spesso clip che mettono in scena le trame relazionali di Hatsune Miku con i suoi amici vocaloid; e addirittura, dal 2013, interprete principale di un'opera ${ }^{3}$. Il successo di Hatsune Miku (il cui numero di fan su Facebook supera oggi i 2,5 milioni) ha trasformato un semplice strumento di marketing in una star peculiare, capace di richiedere a Crypton Future Media, l'azienda all'origine del software e del personaggio, di articolare costantemente la sua comunicazione tra promessa democratica di creazione collaborativa (resa possibile dal programma di sintesi) e ricercatezza evenemenziale di certi show che consolidano la comunità dei fan. In generale, il caso Hatsune Miku può essere analizzato nell'ottica dell'inedita promessa socio-tecnica che esso offre al pubblico; e il caso dell'applicazione di tale meccanismo ad altre star, sia decedute (Tupac, Michael Jackson...) che viventi (Mariah Carey...) o fittizie (Gorillaz), sembra confermarne le molteplici potenzialità - resurrezioniste, ubiquiste... - nella misura in cui rinnova o amplifica alcuni dei nostri interrogativi circa il significato profondo del nostro rapporto con la musica e le sue celebrità. Se i principali sforzi in materia di innovazione musicale si sono concentrati sul miglioramento di dispositivi acustici capaci di restituire un segnale sonoro, la tecnologia dell'ologramma rinnova la "dimensione spettacolare dello spettacolo" accentuando, nel cuore stesso dell'esperienza estetica, ciò che ne costituisce l'istanza più profondamente metafisica, confondendo i limiti tra realtà e illusione, e radicalizzando la questione dell'attaccamento e del desiderio (verso l'opera e verso il suo autore) dal momento in cui essi non sono più rivolti ad un essere in carne e ossa, col suo talento e la sua ispirazione, ma ad una presenza assente o a un'assenza presente. Là dove si incontrano tecnica, industria culturale e nuovi modelli economici, comunità dei fan e pubblico creatore, l'ologramma sembra poter aprire nuove prospettive in campo musicale, prospettive che non si limitano ad un miglioramento significativo dell'esperienza sensoriale - come nel caso del

Miku canta e danza, a volte da sola, a volte in compagnia di altri vocaloid. Crypton Future Media sviluppa così una strategia di marketing cross-mediale, in quanto i brani e le coreografie del gioco noti ai fan di Hatsune Miku vengono poi cantati e danzati in scena, il che certamente contribuisce a rafforzare il senso di attaccamento.

${ }^{3}$ L'opera scritta da Keiichiro Shibuya intitolata The End. 
passaggio da monofonia a stereofonia, o più recentemente con la spazializzazione del suono - ma che creano le condizioni per un movimento riflessivo dell'esperienza estetica stessa, in cui lo spettatore vive sulla propria pelle la mise en abime $e^{4}$ di quella stessa esperienza. È per il suo spostare le frontiere dell'esperienza artistica, e della coscienza che il pubblico ha di tale esperienza, che ci sembra utile considerare le potenzialità artistiche dell'olografia, in particolare tramite il caso di Hatsune Miku.

Dopo aver descritto alcune specificità dell'ologramma di creazione starifié, vedremo come il dispositivo di produzione, diffusione e ricezione di Hatsune Miku, capitalizzando sugli strumenti di creazione che determinano la popolarità della cantante e mettendo in scena (tramite un sistema piramidale di suggerimenti e valutazioni) l'influenza del pubblico sul divenire del personaggio, accentui la logica auto-costitutiva che fa dei mezzi di diffusione al tempo stesso una forma di consumo e un rinforzo della celebrità; logica che si afferma, nel caso di Hatsune Miku, come la condizione stessa di esistenza della star (la cui fragilità costituisce poi una potente risorsa commerciale).

\section{Tipologia degli ologrammi ad uso artistico}

Rendendo visibile un essere privo di presenza corporea, il principio tecnico dell'olografia consiste nel rappresentare un'immagine in tre dimensioni nello spazio reale. Inventata nel 1858 da Henry Dircks e poi adattata al teatro da John Pepper, la prima matrice dell'effetto olografico consisteva in un gioco di proiezioni su una superficie inclinata di $45^{\circ}$ - in tela trasparente o vetro invisibile agli occhi del pubblico, capace di mostrare non direttamente l'immagine, bensì l'immagine dell'immagine proiettata (Pepper 1890). Ne risultava una forma d'illusione ottica impressionante, con immagini fantasmatiche che sembravano fluttuare sulla scena stessa del teatro, dove

\footnotetext{
${ }^{4}$ Espressione inizialmente utilizzata da André Gide, volta ad indicare una strutturazione dell'esperienza su più livelli, legati da un rapporto di ricorsività (tipico il caso del "sogno nel sogno"); si tratta di un espediente narratologico comune alla letteratura, alla fotografia, al cinema (cfr. A. Marchese, Dizionario di retorica e di stilistica, Arnoldo Mondadori Editore, Milano, 1991, p. 203) (N.d.T.)
} 
prendevano vita per poi scomparire ${ }^{5}$. Questo dispositivo per certi versi elementare, ma che ben si adattava al gusto dell'epoca vittoriana per l'aldilà, la metempsicosi e i fantasmi, continuò ad essere sfruttato fino agli anni '60 del Novecento, nei parchi a tema come negli spettacoli teatrali. Tuttavia, si tratta in questo caso di un dispositivo 2D, diverso dall'immagine olografica in senso stretto - la registrazione e la ricostruzione di tutte le informazioni luminose provengono dalla combinazione di un laser e della radiazione diffratta da un oggetto - che suscita la sensazione di una sua effettiva presenza, qualunque sia l'angolo da cui la si osservi. Artisti visivi come Lloyd Cross e Pam Brazier (The Kiss 1973), Paula Dawson (To Absent Friends 1989), John Kaufman (Canted Fragment, 1994), Martina Mrongovius (Pascua Lama, 2006), Ana Maria Nicholson (At the Gate, Cocoon, 2006), Sally Weber (Strata Series 2006) hanno così prodotto opere che testimoniano della grande varietà di dispositivi di generazione olografica ${ }^{6}$.

Se è possibile classificare questi dispositivi olografici secondo il tipo di tecnologia impiegata (Johnston 2016), un secondo principio tipologico rinvia alla natura stessa degli esseri così rappresentati e al tipo di rapporto che ciascuna ontologia è suscettibile di instaurare con il pubblico. A questo riguardo, non stupisce che molti teorici noti per le loro analisi dei legami tra tecnica e modernità (Toffler, Eco, Baudrillard,...) abbiano riservato un posto particolare all'ologramma: Jean Baudrillard, ad esempio, considera l'ologramma non tanto una copia o un'immagine del reale, quanto piuttosto un doppio del suo referente, una forma al contempo iper-realista e iperartificiale, un simulacro. «Nell'ologramma, è l'aura immaginaria del doppio che viene, come nella storia dei cloni, braccata senza pietà» (Baudrillard 1981, p. 156). Più reale del reale, secondo la formula di Baudrillard, all'ologramma mancherebbe, nella sua pretesa iper-oggettivista, ciò che costituisce l'essenza stessa della cosa o dell'essere originario, ovvero la sua parte di ombra, in senso proprio e figurato. E al tempo stesso, come sottolinea lo stesso Baudrillard, iper-similitudine non significa forse uccisione

\footnotetext{
${ }^{5}$ La prima rappresentazione del Pepper's Ghost avvenne nel 1862 a Londra con la messa in scena del racconto di Bulwer Lytton A Strange Story e lo spettacolo The Haunted Man or the Ghost's Bargain di Charles Dickens.

6 Vedi http://www.globalimages-hologramartcollection.com/ http://holocenter.org/artists (ultimo accesso: 20 gennaio 2021).
} 
dell'originale? Da questo punto di vista, l'ologramma andrebbe ad affiancarsi ai campionatori e ad altri dispositivi di digitalizzazione del reale, volti ad assicurare non la riproducibilità tecnica delle cose - e dunque la perdita della loro aura - ma piuttosto la loro riproducibilità infinita e per così dire (quasi) perfetta, in una gradualità sfumata che va dalla copia al clone, dall'uguale allo stesso (l'uno non essendo rigorosamente l'altro, contrariamente a quanto l'espressione quotidiana potrebbe indurci a pensare).

Il potere dell'ologramma risiede innanzitutto nel carattere spettacolare dell'artificio tecnico: un corpo appare, si muove, interagisce con altri corpi affatto reali, come nel caso del festival Coachella 2012 in cui il rapper Tupac - scomparso sedici anni prima - canta e balla con Dr Dree e Snoop Dog. Ciò che colpisce lo spettatore, tanto quanto il riuscito gioco di prestigio, è l'efficienza dell'artificio impiegato: più l'illusione olografica è realistica, più il suo potere di fascinazione si impone a noi. Ma l'impatto dell'ologramma non si riduce alla coscienza dell'artificio tecnico e del suo grado di sofisticatezza: l'ologramma "ripresenta"7 questioni relative alla commensurabilità degli esseri, alla loro vulnerabilità, alla possibilità di un'essenza soggiacente all'apparenza, alla condizione di ciò che è mortale, all'alterità. "What the fucking up, Coachella": nel momento in cui, tutt'a un tratto, Tupac apostrofa il pubblico menzionando un festival che al momento della sua morte non esisteva affatto, non si tratta solamente di un trucco. Se lo spettatore è sbalordito, non lo è tanto per la sofisticatezza dell'artificio tecnico utilizzato, quanto piuttosto per la prospettiva vertiginosa con la quale egli si trova a confronto, a indicare che l'esperienza estetica non si limita all'esperienza del bello o del sensibile, ma si intensifica nel mettere alla prova la sua dimensione più esistenziale, ad esempio laddove essa arricchisce la nostra conoscenza rivelandoci modi inattesi di affrontare il mondo, un cambiamento o un rovesciamento di punti di vista. Cosa accade nel caso del Tupac "resuscitato" dall'ologramma? Da una parte, la sua resurrezione spettrale porta alla luce la questione dell'ambito del possibile, che diviene interrogativo sulla storia e sul senso della storia: cosa sarebbe successo se Tupac non fosse stato ucciso? Avrebbe dato il suo contributo alla storia dell'hip hop, e in quale direzione? In altri termini, l'ologramma di Tupac ci

\footnotetext{
${ }^{7}$ In francese "re-présente" (letteralmente "ri-presenta", ma anche "rappresenta"), gioco di parole intraducibile in italiano.
} 
pone la questione di capire perché le cose stiano così - o se potrebbe, invece, essere altrimenti. Ma, di per sé, non ci offre risposte a tale interrogativo, essendo la semplice riattualizzazione di una forma passata reintrodotta in un presente che è solo artificialmente il suo (ed è divertente veder apparire su Internet commenti sulle scarpe indossate da Tupac a Coachella, vestigia di un'epoca passata). Qui risiede la dimensione "tragica" dell'ologramma, che galleggia sulla superficie di un presente al quale non può tuttavia appartenere, così come galleggia su un palco che i suoi piedi sfiorano senza essere mai perfettamente aggiustati sulla materialità piana e molto concreta della superficie (il che costituisce non a caso una delle sfide principali per $\mathrm{i}$ realizzatori di ologrammi: come far sembrare che essi si muovano "toccando terra"?).

D'altra parte, si ritiene generalmente che un ologramma sia una fotografia in due o tre dimensioni di un essere che gli preesiste. In altri termini, l'ologramma sarebbe rinchiuso nei limiti di ciò che l'essere copiato avrebbe vissuto precedentemente e che sarebbe stato registrato (il che ricalca la trama del romanzo di Adolfo Bioy Casares di cui parleremo poco più avanti: Faustine, di cui il narratore si innamora, recita all'infinito la stessa scena in quanto essa in realtà non è che un'immagine già filmata e poi proiettata incessantemente). L'ologramma sarebbe di conseguenza condannato ad una sorta di eterno ritorno dell'identico, senza possibili scenari alternativi. Tuttavia, lo sviluppo di ologrammi di sintesi sempre più realistici apre la strada a creature autonome, inventate di sana pianta (come nel caso di Hatsune Miku) o ispirate a referenti di cui esse prolungano post mortem l'agire, dando loro, potremmo dire, una nuova vita.

Da questo punto di vista, ci sembra che lo specifico potere di fascinazione dell'ologramma risieda principalmente in due fattori: da una parte, nel fatto che esso getta scompiglio non nell'ambito del genere, per parafrasare Judith Butler, ma in quello dell'ontologia. In altri termini, l'ologramma ci pone la questione di sapere con chi, o meglio, con che tipo di esseri abbiamo a che fare. È davvero Tupac l'ologramma che interagisce con Dr Dree, o è la sua immagine? Una attualizzazione del nostro ricordo di Tupac o un suo rifacimento ideal-tipico? A questo riguardo, occorre senza dubbio operare una 
distinzione tra ologrammi di persone morte, o hantologiques ${ }^{8}$, ologrammi di personaggi viventi e ologrammi di personaggi di finzione: in parole povere, si può dividere $\mathrm{i}$ fenomeni olografici in tre categorie - innanzitutto, gli ologrammi che fanno tornare in scena cantanti morti, come avvenuto per Tupac ma anche per Edith Piaf, Michael Jackson, Elvis Presley, ecc.; in secondo luogo, gli ologrammi che conferiscono esistenza parallela a personaggi reali e viventi, come nel caso di Mariah Carey che si esibì in cinque luoghi contemporaneamente; e infine, gli ologrammi che creano di sana pianta una star a prescindere da ogni referente anteriore, come nel caso dei Gorillaz. In altre parole, ologrammi di riproduzione che conservano nettamente, rinnovandola, la dialettica tra corpo fisico della star (il modello) e le sue riproduzioni (le copie, per quanto esse acquisiscano una particolare indipendenza e un eccezionale livello di realismo); ologrammi di ripresentazione; e infine ologrammi di creazione, che non rinviano più idealmente ad alcun referente corporeo, ma incarnano una star originale creata di sana pianta, come nel caso di Hatsune Miku. Ciascuno di questi tipi di ologramma solleva questioni specifiche: nel caso degli ologrammi hantologiques, si tratta di questioni giuridiche (a chi appartiene il copyright e i diritti d'autore generati dalla performance? Si può far fare "qualunque cosa" a un ologramma, a prescindere da ogni considerazione morale, artistica, ecc.?) e di questioni esistenziali (dove sta il limite tra l'essere e l'apparenza? Come definire qui l'essere e la verità dell'essere?). Gli ologrammi di ripresentazione ci pongono da parte loro la questione dell'hic et nunc: una stessa entità può moltiplicarsi, esibirsi nello stesso momento in contesti differenti e raggiungere quindi un pubblico più vasto, ma anche sottrarsi ai vincoli della prigionia, ad esempio, come nel caso di Julian Assange che partecipò - lui, o meglio il suo ologramma - ad una conferenza negli USA mentre il suo corpo reale era confinato presso l'Ambasciata dell'Ecuador a Londra. Infine, gli ologrammi di creazione sollevano la questione del divenire

\footnotetext{
${ }^{8}$ Il termine francese hantologie (neologismo basato sulla fusione tra ontologie e hanter, infestare), al momento privo di uno stabile corrispettivo italiano, è stato coniato da Jacques Derrida nel suo Spectres de Marx (1993), e si riferisce ad uno stato di ambiguità storicoontologica, la manifestazione di una traccia al tempo stesso visibile e invisibile che "infesta" il presente (N.d.T.).
} 
degli esseri inventati di sana pianta, degli elementi che forgeranno la loro identità e li doteranno di una "natura".

In secondo luogo, se gli ologrammi ci affascinano a tal punto, è per il loro essere al tempo stesso realistici e irreali, e quindi per il loro interrogarci sul tipo di considerazione che possiamo rivolgere loro e di interazioni che con essi possiamo intrattenere. Se ologrammi come Tupac o Hatsune Miku ci scuotono, è perché ci leghiamo ad esseri che possiamo definire esistenti, ma il cui grado o forma di esistenza non corrisponde a ciò che comunemente intendiamo con "esistere". Ne consegue che possiamo affezionarci a loro, provare delle emozioni, addirittura del desiderio nei loro confronti - come succede a numerosi fan di Hatsune Miku - senza tuttavia poter mai accedere ad una interazione vera e propria: in qualche modo, l'ologramma è l'altro nome delle ombre proiettate nella caverna platonica. É questo scollamento, tra attenzione o desiderio per una superficie proiettiva e impossibilità di interagire, che ci interroga al tempo stesso sulla natura dell'attaccamento del pubblico verso la star olografica e sulla coesistenza di due regimi ontologici che si lambiscono senza potersi mai incontrare. Interviene qui la dimensione propriamente spettrale dell'ologramma, che trova il suo principio nella coesistenza di esseri ontologicamente eterogenei, coesistenza che costituisce a sua volta il soggetto stesso di opere come il romanzo L'invenzione di Morel (1940) di Adolfo Bioy Casarès, oppure il film di Robert Zemeckis Chi ha incastrato Roger Rabbit? (1988): nel primo caso, abbiamo una creatura femminile olografica - Faustine - a cui il narratore extradiegetico (Genette: 255) si lega sentimentalmente. Nel suo commento all'opera, Roger Bozzetto (1999: 72) nota a proposito del narratore che «per quanto cosciente dell'impossibilità di incontrare Faustine nella dimensione olografica in cui lei sopravvive come immagine, o persino di farsi conoscere da lei - fosse pure in questa pseudo-vita immaginaria che gli ectoplasmi degli invitati ripetono meccanicamente -, egli spera di avvicinarla». Secondo Bozzetto, la morale della storia è che l'oggetto del desiderio è un'illusione, morale che ci si ripresenta dinanzi agli ologrammi: qual è la natura del desiderio che ci lega alle star o ai loro alter-ego fatti di raggi laser? Nel secondo esempio, invece, come ben riassunto dal critico cinematografico Jonathan Rosenbaum (1988), è «la collisione di due modi di rappresentazione, dotati ciascuno di una opposta relazione con la morte, il sesso, la causalità, la temporalità, 
l'individuo», che genera per il pubblico un certo numero di problemi estetici e ricettivi. Ma è forse il film di Night Shyamalan, Il sesto senso, per quanto non centrato propriamente su ologrammi bensì su fantasmi, che ci sembra tradurre con particolare efficacia questa idea di una eterogeneità irriducibile tra categorie di esseri non coincidenti - eterogeneità d'altronde riconducibile alle relazioni che i fan intrattengono con le star, come giustamente notato da Roland Barthes e Edgar Morin. Nel film di Shyamalan, ciò che conquista il pubblico è precisamente ciò che lo destabilizza, e tutta la genialità de Il sesto senso sta nel semplice fatto che la narrazione ci ancora ad una forma di realismo il cui garante è il punto di vista del narratore stesso: uno psicologo, Bruce Willis, la cui vita cambia pian piano dopo essere stato vittima di un tentato omicidio. Ora, scopriamo alla fine del film, sbalorditi, che questo personaggio che abbiamo visto crescere e (tentare di) interagire con gli altri è egli stesso uno dei morti "vaganti" del film. Gli altri protagonisti non potevano vederlo; solo noi, spettatori, potevamo. Il carattere disturbante di questo film risiede allora nel fatto che, nelle trame di genere simile, il regista offre di solito una chiave di comprensione che sveli razionalmente l'inverosimiglianza della trama: per esempio - soluzione tipica - si potrebbe scoprire che la vicenda era il prodotto di un sogno, che si svolgeva in un mondo di fantasia o che la realtà non era quella immaginata. Ora, nel film di Shyamalan, non c'è nulla di tutto ciò, non c'è un altro livello di realtà capace infine di dare una spiegazione all'inverosimiglianza della trama. Lo spettatore è turbato perché il regista non propone delle alternative: semplicemente non c'è un'altra realtà, la trama non ci ha mentito facendoci credere che Bruce Willis era vivo. Ci siamo, in quanto spettatori, legati ad un essere morto e fantomatico, abitante il mondo dei viventi e lui stesso illuso di poter "pesare" su questo mondo e sul corso delle cose e di poter interagire con i vivi, un morto che non comprenderà di essere morto se non al termine della vicenda.

Uno dei casi più notevoli di iperrealismo olografico e di interazione visivamente riuscita di esseri reali ed esseri virtuali, e proprio per questo disturbante, è quello dell'apparizione di Michael Jackson ai Billboards Music Awards nel 2014 ${ }^{9}$. Circondato da ballerini, il cantante, morto nel 2009, suscita con la sua performance numerose reazioni contraddittorie sul web. Per alcuni, è la presenza assente del cantante defunto a costituire la componente emotiva

\footnotetext{
${ }^{9} \mathrm{https}: / /$ www.youtube.com/watch?v=i-Yys0rhPNQ (Ultimo accesso: 20 gennaio 2021).
} 
dello spettacolo - «This hologram just makes everyone cry... Seeing him on stage thinking he is there but really he isn't» ${ }^{10}$ - laddove altri disapprovano il fatto che si possa provare sentimenti verso un ologramma: «Why are humans so foolish though???? praising a hologram???? really??? wowo.. just wow.... may God help us» ${ }^{11}$. Peraltro, la maggioranza dei commenti si concentra sulla natura incerta di ciò che è dato a vedere, e numerosi sono coloro in cerca di dettagli per confermare che non si tratta né di Michael Jackson, né del suo ologramma, bensì di un semplice imitatore:

- She "Kicks" the Hologram and the Hologram leaves a shadow on her foot. STOP THE VID A 3:04 AND YOU WILL SEE !!! This is not a Hologram, it's an impersonator. She can't kick a hologram and holograms wouldn't leave his legs shadow on her foot.

- I would have to agree, that this looks real and unlike any other hologram that I have ever seen. very creepy.

- Maybe it's not a hologram at all...

- Right double the girl touched his jacket.

- He looks so real like a real person! Is he really just a screen?

- Hmm... i dont know

- The Greatest Entertainer cannot be replaced by a lousy hologram impersonator!! You really believed you had the technology and a stand in under study to equal Michael Jackson ! NEVER !! You LOSE !! ${ }^{12}$

\footnotetext{
10 "Questo ologramma ha fatto piangere tutti...vederlo sul palco, pensare che sia lì ma che in realtà non c'è".

11 "Ma come si può essere così pazzi???? applaudire un ologramma??? veramente???? wow...che Dio ci aiuti"

12 - "Lei "dà un calcio" all'ologramma e l'ologramma lascia un'ombra sul suo piede. FERMATE IL VIDEO A 3:04 E VEDRETE!!! Questo non è un ologramma, è un imitatore. Non può dare un calcio a un ologramma, e un ologramma non proietterebbe un'ombra sul suo piede.

- Sono d'accordo, questo qui sembra reale e molto diverso da qualunque altro ologramma abbia visto. molto inquietante.

- Forse non è affatto un ologramma...

- Confermo, in più la ragazza gli ha toccato la giacca.

- Sembra così reale, come una persona vera! Ma è davvero solo uno schermo?
} 
Potremmo aggiungere, alla lettura di questi commenti entusiasti o disorientati, che un altro punto problematico divide i fan di Michael Jackson: l'ologramma è condannato a svolgere eternamente il ruolo di surrogato rispetto all'originale? $\mathrm{O}$ non potrebbe finire per rivaleggiare con esso, se non superarlo? Se normalmente, con la morte della star, si chiude un'esistenza di cui i biografi provvedevano a portare alla luce ogni frammento di non-detto è così che la morte di Michael Jackson ci ha consentito di accedere a tutta una serie di rivelazioni, mettendo in luce l'interiorità psichica del personaggio e dando una chiave di lettura del suo talento e delle sue scappatelle - il prolungamento hantologique della sua esistenza in forma olografica pone un vero dilemma.

Ad ogni modo, riguardando noi stessi le immagini di Michael Jackson olografizzato sulla scena dei Music Awards, ci sentiamo turbati tanto quanto i fan che si esprimono sul web. Perché?

Senza dubbio perché il talento dell'artista, la vitalità incarnata nei suoi passi di danza, la sua potenza lirica, la sua inimitabile individualità - ciò che alcuni qualificherebbero forse come "genio" se per un momento si accettasse di non ridurre il genio a semplice costrutto sociale - ci si impongono in maniera indiscutibile. L'ologramma di Michael Jackson non suscita, in primo luogo, nostalgia; impressiona in quanto mette in scena il talento come forma pura, o ancora come principio astratto. Se il genio resta nozione difficile da afferrare e fortemente polemica, relativa e, come noto, sfruttata dall'industria musicale e dai media di ogni colore a fini capitalistici, nondimeno resta il fatto che ciò che l'ologramma di Michael Jackson mette in scena è la singolarità essenzializzata di un talento specifico, capace di esistere persino dopo la scomparsa fisica della star. Ciò che ci turba così fortemente è forse allora la doppia natura di questo ologramma: al contempo reificazione di un principio singolare - in questo caso un talento fuori dal comune - in un corpo fatto di particelle luminose, e sua astrazione, sua essenzializzazione. In senso stretto, l'ologramma è compresenza di una cosa e al tempo stesso di un'idea.

- Mmmm... non so

- Il più grande Artista di sempre non può essere rimpiazzato da un misero ologramma imitatore!! Credevate davvero di avere una tecnologia o un sostituto tali da eguagliare Michael Jackson! MAI!! PERDENTI!! 
Ciò che qui vogliamo mostrare, prendendo come esempio Il sesto senso o Michael Jackson, è il fatto che gli ologrammi - proprio come gli spettri - ci turbano e ci affascinano in quanto suscitano in noi emozioni affatto reali, laddove essi non è che non esistano, ma si collocano tuttavia su un piano di esistenza altra. Puri ricettacoli delle nostre emozioni, gli ologrammi di Tupac o di Hatsune Miku sono come uno specchio rivolto ai nostri desideri, ai nostri legami e alle nostre proiezioni, ed è al momento della scomparsa dell'ologramma - spesso un improvviso disintegrarsi del corpo sotto i nostri occhi in una nube di scintille luminose - che ci torna alla mente non tanto l'artificio reso possibile dal miracolo tecnologico, quanto piuttosto la sua alterità radicale e il suo potere di articolare arte e metafisica.
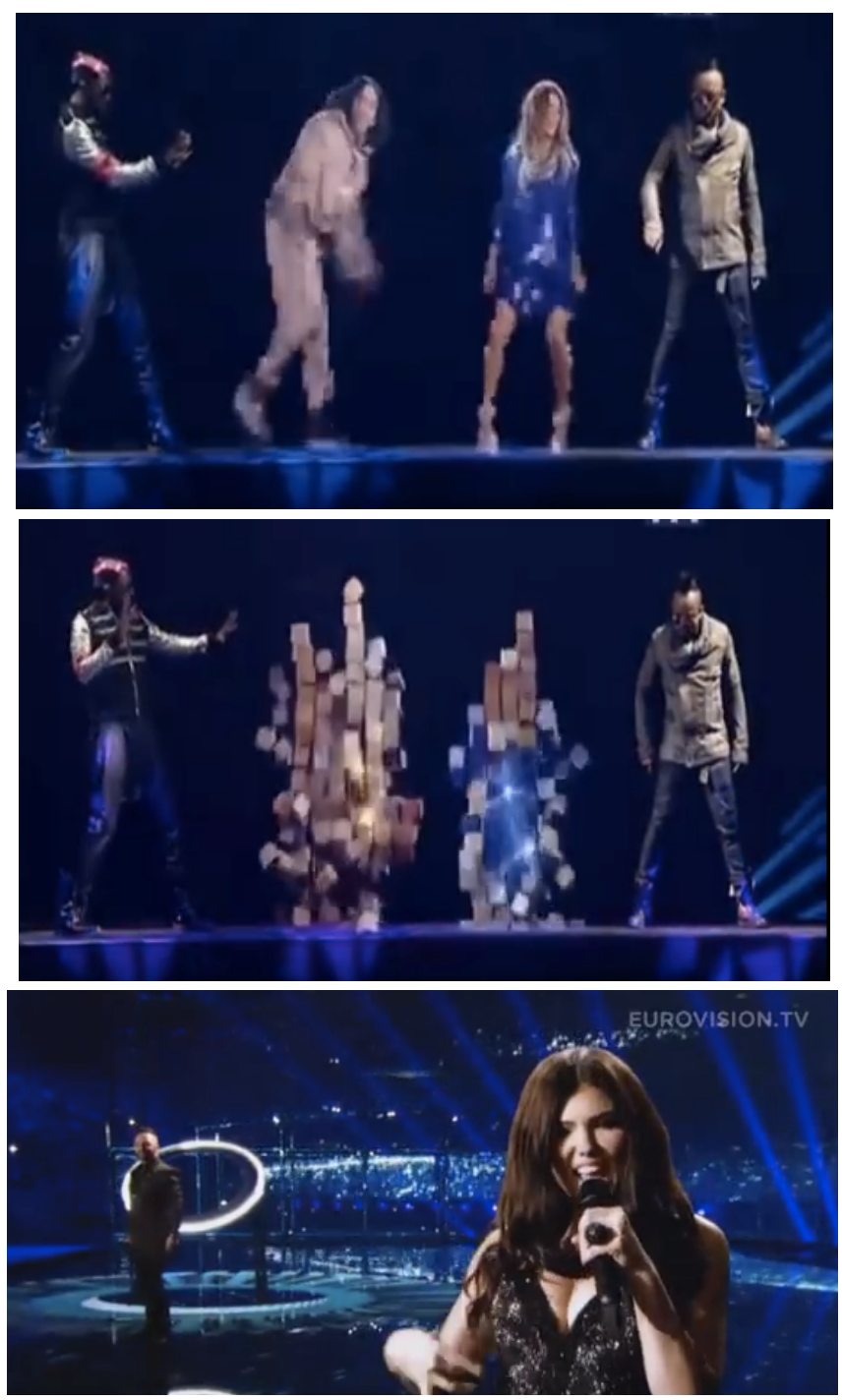

Fig. 1 Alterità radicale e nascondimento dell'essere (fonte : Youtube) 


\section{Il caso Hatsune Miku}

A differenza degli ologrammi che riportano in scena per qualche ora una star scomparsa o di quelli che moltiplicano l'immagine di una ancora viva per accrescere l'illusione della sua ubiquità, l'immagine olografica di Hatsune Miku non rinvia ad alcun referente anteriore. Non riproduce un corpo vivente, un originale a cui l'ologramma rimanderebbe in quanto copia, ma costituisce essa stessa il referente - che crea nello stesso momento in cui lo diffonde. Questa differenza di formato, che apre ad uno specifico regime di finzione e che colloca Hatsune Miku a metà strada tra il personaggio di fantasia e la popstar, cambia il rapporto tra l'ologramma e la famosa spettralità della registrazione ${ }^{13}$, vale a dire l'idea seconda la quale, come formulato da Jacques Derrida in Ecografie della televisione, lo sdoppiamento della cattura fa immediatamente del corpo registrato uno spettro pensato come «traccia che marca in anticipo il presente della sua assenza» (Derrida \& Stiegler, 1997: 131). Perché se gli ologrammi di riproduzione spingono all'estremo questo processo di sdoppiamento (nostalgico o ubiquitario), ne conservano pur tuttavia la dialettica di base (poggiata sul rapporto tra il corpo fisico della star e le sue riproduzioni), laddove gli ologrammi di creazione, escludendo la star da qualunque vita fuori dalla rappresentazione, ripensano il legame tradizionale tra presenza straripante di immagini e inaccessibilità ${ }^{14}$.

\subsection{L'immagine evanescente}

Al tempo stesso sintesi vocale e animazione 3D, Hatsune Miku è dunque concepita come una superficie proiettiva particolarmente performante: da un lato, essa si presenta come un'immagine o una rappresentazione priva di intenzione, pronta a interpretare entro i limiti tecnici del programma tutto ciò che l'utente desidera farle cantare; dall'altro, essa recupera la propria

\footnotetext{
${ }^{13}$ Cfr. tra gli altri, su questo punto, Reynolds 2011.

${ }^{14}$ È questa articolazione che, secondo Nathalie Heinich, definisce il «detentore di un capitale di visibilità»: «inaccessibile di persona e al contempo indefinitamente disponibile nelle sue immagini» (Heinich 2012, p. 453).
} 
autonomia e si impone nella sua individualità, durante i grandi concerti, di fronte alla folla anonima del pubblico. ${ }^{15}$

Il successo di Hatsune Miku si poggia su questa tensione, in quanto essa permette di conservare la passione del fan per la star (che resta intoccabile) e al tempo stesso mobilitare l'attivo coinvolgimento dell'ascoltatore (al quale è affidato il compito di far "vivere" la cantante). Il dispositivo preserva insieme l'illusione della prossimità e l'asimmetria del rapporto: il fan può partecipare personalmente alla produzione di Hatsune Miku, può farla cantare e ballare, ma essa resta, letteralmente, inafferrabile. È quindi al contempo inaccessibile, riproducibile e manipolabile.

Crypton Future Media mette in scena questo paradosso, insistendo simultaneamente sulla disponibilità di Hatsune Miku (la cui voce e il cui corpo modellizzato sono in vendita sul sito dedicato per poco più di un centinaio di euro) e sulla rarità dei concerti. Da questo punto di vista, colpisce che l'ologramma che canta e balla in scena - mera immagine tra immagini sia stranamente proposto e vissuto come il modello da cui deriverebbero tutte le altre immagini: vedere Hatsune Miku in concerto, vedere il suo ologramma sulla scena acquista un valore specifico, paragonabile a quello che possono avere i concerti dei gruppi rock e pop tradizionali. Questa costruzione della singolarità del live ha dunque l'effetto di elevare un'immagine (l'ologramma) allo statuto di modello. Tutto, durante i concerti, mira ad accentuare l'effetto di fragile presenza di Hatsune Miku, sia accentuando la discontinuità ${ }^{16}$, sia simulando un vissuto emozionale condiviso da personaggio e fan. È il caso di quanto successo nel 2011, ad esempio, in occasione di un grande concerto in Giappone durante il quale Hatsune Miku ha attaccato una canzone, dolce e triste, When the First Love Ends, poi, la voce tremante come se fosse

\footnotetext{
${ }^{15}$ Sulla «alleanza di iper-individualizzazione della star e di iper-massificazione della folla», cfr. Heinich 2012, p. 178.

${ }^{16}$ Hatsune Miku compariva e scompariva spesso per dissolvenza progressiva o in una nuvola di pixel, come all'inizio di The Intense Singing of Hatsune Miku (https://www.youtube.com/watch?v=WJV6aXhehrk) (ultimo accesso: 20 gennaio 2021) o alla fine di The Disappearing of Hatsune Miku (https://www.youtube.com/watch?v=9LG7wo82ivs: Ultimo accesso: 20 gennaio 2021).
} 
assolutamente viva e sensibile, dopo pochi secondi si è fermata e si è asciugata una lacrima sulla guancia, prima di ricominciare a cantare ${ }^{17}$.

Assenti per definizione dall'immagine olografica, tutte le coordinate spazio-temporali del corpo - come l'unicità di luogo e di tempo, l'invecchiamento, gli accidenti, le variazioni e le incertezze espressive - si trovano dunque, nel caso di Hatsune Miku, o, come appena visto, esacerbate artificialmente al momento della rappresentazione (per tentare di rafforzare il senso di personificazione dell'ologramma), oppure esplicitamente eluse: in nove anni di esistenza, Hatsune Miku non ha preso una ruga, restando, dalle sue prime apparizioni, l'adolescente di 16 anni le cui modifiche nell'aspetto sono dovute piuttosto a ragioni commerciali (come la presentazione di una creazione originale targata Vuitton) che a trasformazioni corporee. Così, Hatsune Miku cristallizza un effimero infinitamente riproducibile, legando assieme una inalterabilità di principio e una «posizione di evanescenza», per riprendere la formula di Barthes (Barthes 1957, p. 27).

\subsection{Dispositivo di ascolto e valutazione}

Le diverse tensioni appena riassunte si ripercuotono ovviamente sulla ricezione delle produzioni di Hatsune Miku, ricezione le cui specificità appaiono chiaramente in seno al confronto tra fan sui numerosi forum dedicati alle discussioni e alle valutazioni dei brani interpretati dalla cantante olografica. Tali specificità risiedono in primo luogo nella grande varietà stilistica di Hatsune Miku, il cui repertorio considerevole ${ }^{18}$, composto tanto da pezzi originali scritti appositamente per lei quanto da cover, copre una quantità di generi certamente ineguagliata da una singola interprete: innanzitutto il pop, ovviamente (che costituisce la gran parte del repertorio), ma anche rock, rap, elettronica, neo-metal, dubstep, reggae, dance, arie classiche, e numerosi successi come quelli di Lady Gaga, Céline Dion, Madonna, canzoni di Gershwin, Brassens o Joe Dassin... Tanto che essere fan di Hatsune Miku non significa essere legati ad un genere particolare o definire il proprio gusto secondo criteri di distinzione stilistica, ma vuol dire

\footnotetext{
17 Cfr. https://www.youtube.com/watch?feature=player_embedded\&v=0-tQIMG-CqA. (Ultimo accesso: 20 gennaio 2021). Questa emozione simulata ha sconvolto la comunità dei fan.

${ }^{18}$ Stimato a più di centomila brani.
} 
piuttosto ammirare un "concept" (termine spesso suggerito dagli stessi fan ${ }^{19}$ ), una voce e la stessa tecnologia che li rende possibili. Nelle discussioni, assai apprezzate sono d'altronde la capacità di analisi dei pezzi in funzione di criteri specifici a ciascun genere e la tolleranza rispetto alla diversità di produzioni, essendo un brano solo raramente, stando al panorama dei forum che abbiamo potuto considerare, discriminato a priori per motivi stilistici ${ }^{20}$.

Questa necessità di analisi riposa sul principio stesso della diffusione di brani che, nell'apparente orizzontalità del web, si sviluppa di fatto su più livelli, secondo un'architettura piramidale, che spazia dalla molteplicità dei forum dei fan (sui quali sono postati tanto i tentativi di principianti che tentano di padroneggiare il programma, quanto quelli di semi-professionisti che testano le loro canzoni nella prospettiva di realizzare un album ${ }^{21}$ ) fino alle grandi classifiche internazionali ${ }^{22}$. Se nel primo caso i prodotti della comunità dei fan, limitati ma nondimeno oggetto di grande impegno, testimoniano una certa eterogeneità, nel secondo caso, basato più su voti e valutazioni che su commenti e consigli, le produzioni hanno maggiore uniformità. Infine, a coronare questo già variegato repertorio, arrivano le commissioni o le

19 Cfr. ad esempio il commento di Aono in risposta al topic «Perché apprezzate VOCALOID?»: «Personalmente, mi metterò forse in cattiva luce, ma preferisco il concept VST di Vocaloid rispetto alla musica che con esso ci viene proposta» (http://miku.vocaloid.fr/index.php/topic,4434.0.html) (Ultimo accesso: 20 gennaio 2021).

${ }^{20}$ Piuttosto che l'affermazione di regole estetiche generali, questa apertura richiede quindi delle micro-analisi: il suono della chitarra adatto ad un pezzo rock non si presterà per un brano più pop, il mixaggio della voce in stile metal sarebbe terribile in un pezzo più dolce, ecc... Uno dei criteri più determinanti nell'apprezzamento di un brano è il mixaggio della voce - intonazione, compressione, vibrato ed effetti (cfr. ad esempio: miku.vocaloid.fr/index.php/topic,3228.105.html).

${ }^{21} \mathrm{Cfr}$. ad esempio un forum molto attivo: http://miku.vocaloid.fr/

${ }^{22}$ Come Mikubook.com che, tra il 2011 e il 2015, proponeva una classifica internazionale di brani di Hatsune Miku. Occorre notare che, nel senso dell'apertura stilistica sopra evidenziata, Mikubook non classificava per generi ma per aree geografiche e tag riguardanti principalmente personaggi, tematiche delle canzoni o caratteristiche informative come remix, brano originale, ecc... Si passava dunque, restando sull'ascolto dei brani in testa alle classifiche top 10 o top 20, da un genere all'altro. 
collaborazioni ufficiali, in genere prodotte da Crypton Future Media ${ }^{23}$. Dalla creazione amatoriale e partecipativa alle superproduzioni pop, ciascun livello di ascolto non è eccezionale in sé, ma la loro unione in una sola medesima interprete, capace di coinvolgere i generi musicali più disparati, costituisce un tratto peculiare di Hatsune Miku. Ora, questo dispositivo comprende la già descritta articolazione di singolo e molteplice, in quanto l'ascolto del fan, tramite un sistema di voto e selezioni, va in parte a costruire in anticipo il successo musicale, in modo tale che la democratizzazione rivendicata da Crypton Future Media diventi duplice - concernente tanto la produzione quanto la ricezione. Non solo ogni acquirente del software può comporre per Hatsune Miku, ma ogni ascoltatore può, in una certa misura, scegliere i successi che andranno a definire la rappresentazione della sua star per il grande pubblico. L'ascolto diventa quindi, nello stesso dispositivo di diffusione, un modo di costruire il repertorio; esso è pensato come un elemento concreto di elaborazione dell'identità in divenire dell'ologramma.

Questa valorizzazione dell'ascolto nella sua dimensione al contempo collettiva e selettiva suppone un sistematico sforzo argomentativo, richiesto in maniera ricorrente sui forum ${ }^{24}$. I commenti sulle canzoni alternano così descrizioni musicali miste a impressioni - la tale canzone «ci perde in un vortice di rytHatsune Miku molto rapidi e molto lenti, soffocandoci al limite dello sgradevole, per poi ritrovare, sprofondati in questo caos, la vera armonia, recuperando la calma con perseveranza $»^{25}-$ a critiche tecniche con consigli molto più precisi ${ }^{26}$. Queste analisi sono spesso inframezzate da

\footnotetext{
${ }^{23} \mathrm{Cfr}$. ad esempio, su questo punto, l'intervista di Eiji Hirasawa, il compositore che ha scritto il primo brano destinato ad Hatsune Miku, su commissione di Crypton Future Media: https://www.youtube.com/watch?v=91fPseUzzJs

24 Cfr. ad esempio http://miku.vocaloid.fr/index.php/topic,1453.msg178622.html\#msg178622: «Non ve lo diciamo mai abbastanza ma A-R-G-O-M-E-N-T-A-T-E su questo topic. Una sola frase non è sufficiente bisogna davvero sostenere questa canzone, parlare del suo produttore diciamo, e spiegare veramente PERCHE' questa canzone e non un'altra».

${ }^{25} \mathrm{http} / / /$ miku.vocaloid.fr/index.php?topic $=1453.170$

${ }^{26} \mathrm{Cfr}$. ad esempio http://miku.vocaloid.fr/index.php/topic,3228.105.html :

«Ecco i miei consigli:
} 
spiegazioni o giustificazioni del fan riguardo il proprio attaccamento a Hatsune Miku, giustificazioni tra le quali emerge principalmente la sfida tecnica e il funzionamento collaborativo associati alla $\operatorname{star}^{27}$, affermando alcuni di preferire esplicitamente il dispositivo e la novità di Hatsune Miku rispetto ai prodotti concreti a lei associati ${ }^{28}$.

- Niente sidechain compression per il mixaggio, è solamente per fare un effetto, beh io non penso che tu l'abbia utilizzato ma talvolta fanno sidechain sullo strumento in rapporto alla voce, ma francamente lo trovo orribile.

- Niente compressione su "gruppi di strumenti”, dà l'impressione che gli strumenti si soffochino a vicenda.

- $\quad$ Per il beat, raggruppa per "altezze", ho trovato che funzioni bene, un compressore per tutto ciò che è $\mathrm{HH}$, piatti e compagnia, un compressore per snare e rimshot, un compressore per la grancassa ecc. I bassdrum hanno di solito la loro propria compressione perché bisogna davvero stare attenti con i bassi.

- $\quad$ Metti un (1) compressore sul master. Sul mio ultimo pezzo ne ho messo uno per gli alti e uno per i bassi (quindi alla fine il segnale passa per un (1) compressore!), e ho variato i parametri, invece il gain resta lo stesso sennò fa una cosa orribile, mi sembra che abbia funzionato bene, puoi ascoltare per giudicare. Invece visto che hai la voce, penso che dovresti separare il segnale della voce (quella principale in ogni caso) e mettergli un comp e un limiter dedicati.

- $\quad$ Io metto un (1) limiter alla fine del master, ma non serve a granché visto che anche senza il mio pezzo suona già molto bene. Se il pezzo è clippato ovunque prima del limiter, si allunga troppo e fa schifo, il limitatore serve infatti a tagliare là dove si esagera veramente ad esempio quando hai tutti gli strumenti che ritornano insieme con un colpo di BD e un colpo di piatti, chiaro l'esempio?

- $\quad$ Filtri! Metti filtri ovunque! Sembra strano ma un suono filtrato si sente meglio! Ho notato che succede soprattutto con i suoni brevi (beat) ad esempio quando filtro lo snare ho l'impressione che guadagni decibel. Ti consiglio di filtrare i suoni ma di smettere non appena si sente, filtri senza che si senta, sembrerebbe un lavoro inutile ma alla fine il mix guadagna in volume».

27 Cfr. http://miku.vocaloid.fr/index.php/topic,4434.0.html. Ultimo accesso: 20 gennaio 2021.

${ }^{28}$ E' il caso già citato di Aono: «È l'idea di Vocaloid in quanto strumento che mi attrae. Amo la musica molto contemporanea e apprezzo l'innovazione in campo musicale. Un VST vocale mi è parso molto interessante. Poi, a proposito dell'universo e delle musiche prodotte a livello ufficiale, bah... Sono alquanto titubante. Adoro i manga e il Giappone, non è quello che mi 
"Disegnatori", "ballerini”, “scrittori”, "modellatori”, "animatori”,
"pittori”, "calligrafi”, "scultori”, "webmaster" e "programmatori" 29 la molteplicità di talenti che costituisce la comunità dei fan, puntualmente messa in evidenza, si esprime in particolare attraverso le clip che costituiscono il vettore privilegiato per la diffusione dei brani e che si impone come la dimensione più diffusa dell'opera, tanto che la musica stessa diviene spesso un semplice parametro ${ }^{30}$ tra gli altri nei criteri di valutazione.

Infine, se non si può propriamente parlare di "mediatizzazione"31 (non essendoci una vita intima della star), esiste d'altronde, a partire dagli aneddoti d'infanzia fino alla vita affettiva e sentimentale, una esistenza fittizia molto ricca di Hatsune Miku, in gran parte elaborata dagli stessi fan nelle light

disturba. Tuttavia, mi piacerebbe che i Vocaloid fossero più aperti ad altre atmosfere musicali. I produttori si accontentano troppo del Jpop facile, a volte dell'elettronica, ma sempre chiusi in questo circuito manga-giapponese che penetra male da noi, ma che è molto accessibile da loro. Di fatto non ascolto molte canzoni vocaloid. Ne apprezzo qualcuna, ma sono molto selettivo. Spero che un giorno lo strumento Vocaloid si aprirà a nuovi orizzonti musicali più sperimentali, ed è compito degli artisti pensarci (ringrazio già London Elektricity per aver utilizzato Vocaloid in uno dei loro singoli, ma non è ancora sufficiente). Bisogna prendersi dei rischi, insomma».

(http://miku.vocaloid.fr/index.php/topic,4434.0.html: ultimo accesso: 20 gennaio 2021).

${ }^{29}$ Questa lista si ispira a un post di Luccar25: «potete voi stessi constatare che VOCALOID non riguarda soltanto musicisti (produttori di musica o autori di cover strumentali, cantanti,...), per quanto essi siano già assai numerosi per conto loro. Ci sono anche i disegnatori, che sono, credo, più numerosi dei musicisti. Aggiungiamo anche i ballerini! Ma non solo loro! Anche scrittori, che producono light novels, artisti che producono animazioni, in $2 \mathrm{D}$ o $3 \mathrm{D}$. E per i modelli $3 \mathrm{D}$, sono servite delle persone per crearli. Si possono poi aggiungere pittori, calligrafi, e anche scultori, chi crea i modellini. C'è persino un progetto di Anime che la segue in questo preciso momento! E si può aggiungere alla lista la creazione di siti web e di comunità sul web. Senza dimenticare i programmatori che creano il programma e le banche dati vocali».

(http://miku.vocaloid.fr/index.php/topic,4434.0.html: ultimo accesso: 20 gennaio 2021)

30 Ad esempio, su http://miku.vocaloid.fr/ (ultimo accesso: 20 gennaio 2021), la rubrica musica è semplicemente una delle sotto-rubriche della rubrica “creazioni” che raggruppa clip, traduzioni, grafica, scrittura, foto...

31 "Peoplisation" nell'originale, che è termine francese più specifico nell'indicare la mediatizzazione della vita pubblica di una celebrità. 
novels, nelle canzoni e nelle clip. Lungi dal formare un tutto coerente, queste storie costruiscono piuttosto una seria di mondi paralleli in costante modulazione. Non appena ci si interessi ad Hatsune Miku, ci si trova al cospetto di una pluralità di costruzioni biografiche che divergono le une dalle altre. ${ }^{32}$ Ora, questa parte di finzione connota fortemente l'ascolto in quanto focalizza l'attenzione sulla narrazione, sulla costruzione aperta del personaggio, e dà luogo a numerose controversie ermeneutiche, quali le speculazioni e i dibattiti elaborati in merito alla relazione amorosa tra Hatsune Miku e Kaito (il primo vocaloid maschio), relazione la cui indeterminatezza lascia campo libero a dicerie e proiezioni di ogni genere: se in una clip si vede un poster di Len, un'altra vocaloid ugualmente creata nel 2007, sulla parete della stanza di Kaito - come avviene in Rotten Girl, Grotesque Romance - la sfida diventa allora determinare se quell'indizio sia davvero la "prova" che Len e Kaito hanno una relazione ${ }^{33}$. Parte dell'attenzione può quindi fissarsi su questo genere di dettagli visuali delle clip o sui sottintesi dei testi per decrittarli, per speculare sulle loro conseguenze o anche per giudicare moralmente il comportamento dei personaggi, il che dà luogo talvolta a confidenze intime e a consigli tra fan su questa o quella scena di vita quotidiana.

Questa aggiunta di una superficie proiettiva, di una dialettica dell'inaccessibilità e della creazione, di una costruzione fittizia ramificata e frammentaria di Hatsune $\mathrm{Miku}^{34}$ come di un carattere collaborativo della sua produzione e della sua ricezione, tutto ciò va a costruire dunque una star a metà strada tra il personaggio manga e la cantante pop, e provoca, in questo

\footnotetext{
${ }^{32}$ Le storie parallele sono numerose: nella canzone Kimi Boshi, ad esempio, Hatsune Miku muore giovane quando sarebbe voluta diventare una cantante (https://www.youtube.com/watch?v=4qNvUrN9h14; ultimo accesso: 20 gennaio 2021) e tutta una mitologia si costruisce su questo desiderio di gloria infranto...

${ }^{33} \mathrm{Cfr}$. https://www.youtube.com/watch?v=eiW9nPFWhV4\&lc=rQmJnQQx-YquLh6uQWWAd617UeY-yKxB15qPh7r0Ww (ultimo accesso: 20 gennaio 2021).

${ }^{34}$ Certi album costruiscono ugualmente, di canzone in canzone, trame più lunghe dove le piste narrative si legano tra un brano e l'altro, dipanando o infittendo le trame. È il caso ad esempio di Wowaka con Unhappy refrain (https://www.youtube.com/watch?v=v36KMdiWHao: ultimo accesso: 20 gennaio 2021).
} 
mescolarsi di potenti effetti di identificazione presso i fan ${ }^{35}$ la cui ricezione articola giudizi estetici e morali, apprezzamenti tecnici, interpretazioni narrative e considerazioni generali sulle prodezze tecnologiche o il sentimento di appartenenza a un movimento originale. È su questa interfaccia tra finzione e divizzazione che l'evoluzione del personaggio si dispiega da diversi anni, sviluppando gradualmente, e in opposizione ad una prima immagine molto gioiosa, colorata e kawai in gergo (carino in giapponese), un versante assai più oscuro che mette Hatsune Miku di fronte alla violenza, a forme di perversione o di distruzione. L'opera di Keiichiro Shibuya intitolata The End, realizzata allo Chatelet nel novembre 2013 e inserita in questo percorso di incupimento del personaggio, mette precisamente in scena quel nodo cruciale su cui si dispiega la celebrità di Hatsune Miku: il libretto descrive, in effetti, la presa di coscienza da parte della star della propria condizione di ologramma, dei limiti del suo proprio ascolto che diviene generica incarnazione dei suoi limiti esistenziali. Sin dalla prima scena, Hatsune Miku è accompagnata da un compagno che costituisce come una protesi sensibile e che commenta tutto ciò che accade, cercando in particolare di decrittare i messaggi diffusi dagli altoparlanti e coperti dai rumori della città in cui si trovano i due protagonisti. Rapidamente, un doppio umano di Hatsune Miku si presenta all'ologramma, rivelandogli la sua finitezza. Contrariamente al motivo classico fantascientifico delle super capacità degli umanoidi, in questo caso non è la capacità di ascolto sovrumana dell'ologramma che viene esibita, bensì una forma di tragedia, che si appoggia sull'idea che il solo interprete dell'opera non sia in grado di

\footnotetext{
${ }^{35}$ La pregnanza di questo universo di finzione ramificato e frammentario, che possiamo costruire, interpretare e giudicare, è tale che gli effetti di riduzione sulle incoerenze narrative sono assai rari e che in certi commenti Hatsune Miku sembra esplicitamente trarsi fuori dalla finzione come in questa considerazione di LioChanDaYo a proposito dell'opera The End: «La paura di morire di un soggetto virtuale a seguito della presa di coscienza della sua esistenza, ammetterai che non è cosa che si vede tutti i giorni, soprattutto in questo caso preciso, dove il soggetto virtuale preesiste alla trama - dopo tutto, Miku potrebbe tranquillamente prendere coscienza della sua esistenza nel nostro mondo - e chi ti dice che non sia questo il caso? (...) il personaggio esiste nel nostro mondo [sottolineato da LioChanDaYo] da 6 anni. Non è più un personaggio di finzione». (http://forum.nolifetv.com/showthread.php?p=2149883; ultimo accesso: 20 gennaio 2021).
} 
ascoltarla... Hatsune Miku finirà per morire della presa di coscienza della propria condizione (per quanto ologramma, non è infatti eterna), con un'ultima canzone dove essa ripete, in una frase continua che si rinchiude su se stessa: "non sento nulla, non vedo nulla, non provo nulla, non sento nulla, non vedo nulla, non provo nulla...". Come se il personaggio vivesse, nell'opera, lo sgretolarsi della parte di credenza sulla quale riposa, nella realtà, tutta la costruzione dei sistemi d'ascolto gerarchizzati che gli danno forma.

[Tradotto dal francese da Ruben Marzà]

\section{Bibliografia}

BARTHES R. (1957), Mythologies, Seuil, Paris.

BAUDRILLARD J. (1981), Simulacres et simulation, Galilée, Paris.

BOZZETTO R. (1999), L'invention de Morel. Robinson, les choses et les simulacres, «Études françaises», 35, 1, pp. 65-77.

DERRIDA J., STIEGLER B. (1997), Echographies de la télévision: Entretiens filmés, Galilée, Paris.

GENETTE G. (1972), Figures III, coll. «Poétique», Seuil, Paris.

HEINICH N. (2012), De la visibilité. Excellence et singularité en régime médiatique, Gallimard, Paris.

JOHNSTON S. F. (2016), Holograms: A cultural history, Oxford University Press, Oxford.

PEPPER J. (1890), The true history of the ghost: And all about metempsychosis, Cassell, London.

REYNOLDS S. (2011), Retromania. Pop culture's addiction to its own past, Faber and Faber, London. 